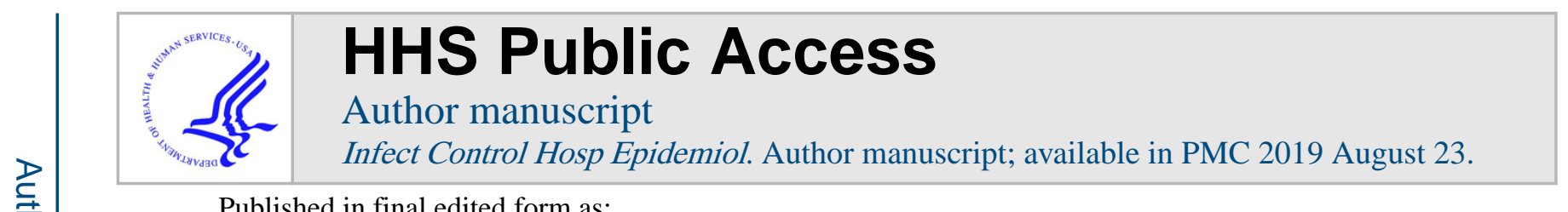

Published in final edited form as:

Infect Control Hosp Epidemiol. 2015 December ; 36(12): 1477-1478. doi:10.1017/ice.2015.232.

\title{
Reply to Jones et al
}

\author{
Minn M. Soe, MBBS, MPH, Carolyn V. Gould, MD, MSCR, Daniel Pollock, MD, Jonathan \\ Edwards, MStat \\ Division of Healthcare Quality Promotion, Centers for Disease Control and Prevention, Atlanta, \\ Georgia.
}

\section{To the Editor-}

We appreciate the insightful thoughts and excellent comments by Jones et al, ${ }^{1}$ and we are grateful to the journal editors for the opportunity to respond.

Healthcare metrics used to guide prevention depend upon the goals of the efforts and should facilitate implementation of strategies at multiple levels, from the local unit to state, regional, and national efforts. We agree that the cumulative attributable difference (CAD) metric is "only part of the puzzle" in improving healthcare quality, as pointed out by Jones et al. ${ }^{1}$ The CAD was developed as a part of the Centers for Disease Control and Prevention's Targeted Assessment for Prevention (TAP) strategy to facilitate using data for action by targeting areas of need and operationalizing standardized infection ratio (SIR) goals into actionable, quantifiable prevention targets. ${ }^{2}$ The $\mathrm{CAD}$, if positive, is the number of healthcare-associated infections (HAIs) that must be prevented to reach a desired SIR goal in a defined period. In addition, the CAD can be useful in targeting areas, particularly at the unit level, that need improvement but might otherwise be overlooked.

As part of a multifaceted approach to measuring and reducing HAIs, the CAD should be used in conjunction with the $\mathrm{SIR}^{3}$ and other information needed to guide decisions. State partners are using innovative approaches, including the TAP strategy, to identify and address gaps in infection control across networks of facilities. For example, the Tennessee Department of Health is accessing data reported to the National Healthcare Safety Network (NHSN) and using analytic methods that include both CAD and SIR metrics to target healthcare facilities where patients presenting to the hospital from the community or other facilities, such as nursing homes, account for a disproportionate burden of Clostridium difficile infections. ${ }^{4}$ Using this approach, Tennessee is strategically working toward state HAI prevention goals by addressing the problem across the continuum of care.

Jones et al ${ }^{1}$ state that "from a healthcare network perspective, it is conceivable that it may be more costly to prevent a single additional infection in a large hospital with a moderate HAI rate than in a small hospital with a high HAI rate. As a consequence, the potential impact of an intervention (the count of HAIs prevented) may not correlate with CAD." This statement

Address correspondence to Minn M. Soe, MBBS, MPH, MS A-24, CDC, 1600 Clifton Rd NE, Atlanta, GA 30333 (msoe@ cdc.gov). 
suggests a role for the $\mathrm{CAD}$ - evaluating the potential impact of interventions-that goes beyond our proposed use of the CAD to identify and prioritize prevention targets on the basis of previously reported HAI data. Cost considerations almost inevitably enter into decisions about what prevention efforts to initiate, and although cost is an important determinant of action, the CAD aims to provide data about opportunities for intervention that might otherwise be overlooked and as a result never entered into a cost-benefit analysis. The CAD metric is not intended to measure outcomes or to predict either the CAD prevented or the costs of a given strategy. In the article, ${ }^{2}$ we also cautioned that the CAD should not be used as a comparative metric among hospitals for public reporting or other purposes outside of quality improvement, because the CAD is not adjusted for risk exposure volume (eg, catheter-days). We emphasize the importance of continuing to use comparative metrics such as the SIR or incidence density rates to monitor the impact of prevention strategies and continue to improve healthcare quality. We agree that more research is needed to improve the prediction of cost savings across the variety of HAIs that could be targeted and prevented.

The CAD tends to identify areas with higher exposure volume of risk (larger hospitals), as illustrated in Table 1 of our article. ${ }^{2}$ However, the CAD also helps to identify and respond to prevention opportunities that might otherwise be missed. For example, a small hospital or unit may not have a SIR computed because the predicted number is less than 1, resulting from low exposure volume of risk, but still have a positive CAD that would indicate a need for attention. Alternatively, a large hospital with high exposure volume may have a large burden of infections reflected in the CAD despite having a low or moderate SIR, which might be overlooked.

The CAD already is available for use through the TAP report analytic function built into the NHSN of the Centers for Disease Control and Prevention. The TAP reports summarize multiple data points from a single hospital or a group of hospitals reporting to NHSN into a single report, including SIR, device utilization, and pathogen data. ${ }^{5}$ Through TAP reports, NHSN users can identify areas of patient care that signal a need for increased focus on specific HAI prevention. Therefore, a facility, healthcare system, or quality improvement organization can target resources to units with high excess burden of HAI (prioritarianism) and will have an overall impact on the facility or group SIR (utilitarianism). As of August 6, 2015, NHSN hospitals, including long-term acute care and inpatient rehabilitation facilities and group users, have run more than 17,000 TAP reports. Currently, we are collecting data to evaluate the impact of the TAP strategy on process and outcomes measures.

In summary, the CAD metric helps to focus prevention efforts and complements the SIR. The TAP strategy and CAD metric have been well received by state public health partners working on HAI prevention because the CAD is conceptually easier to understand than the SIR and drives action, based on an assessment of infection prevention gaps in targeted areas, toward a desired SIR goal. We appreciate the insights and discussion by Jones et $\mathrm{al}^{1}$ as we continue to develop methods to further augment prevention efforts. 


\section{ACKNOWLEDGMENTS}

Financial support. None reported.

Disclaimer: The findings and conclusions in this report are those of the authors and do not necessarily represent the official position of the Centers for Disease Control and Prevention.

\section{REFERENCES}

1. Jones MM, Nelson RE, Rubin MA. Cumulative attributable difference: an infection metric that reflects a value system. Infect Control Hosp Epidemiol 2015.

2. Soe MM, Gould CV, Pollock D, Edwards J. Targeted assessment for prevention of healthcareassociated infections: a new prioritization metric. Infect Control Hosp Epidemiol 2015.

3. Centers for Disease Control and Prevention. National Healthcare Safety Network (NHSN) e-news: SIRs special edition. CDC website. http://www.cdc.gov/nhsn/PDFs/Newsletters/ NHSN_NL_OCT_2010SE_final.pdf. Updated 12 10, 2010 Accessed September 12, 2015.

4. Centers for Disease Control and Prevention. Vital signs: estimated effects of a coordinated approach for action to reduce antibiotic-resistant infections in health care facilities-United States. MMWR Morb Mortal Wkly Rep 2015;64:826-831; http:/www.cdc.gov/mmwr/preview/mmwrhtml/ mm6430a4.htm?s_cid=mmmm6430a4_w. Accessed September 12, 2015. [PubMed: 26247436]

5. Centers for Disease Control and Prevention. The five "W"s of the targeted assessment for prevention (TAP) strategy. CDC website. http://www.cdc.gov/hai/prevent/tap.html. Accessed September 12, 2015. 